\title{
Equations of Prolate Spheroid in Spherical Polar Coordinates and its Surface Area by Analytical Method
}

\author{
Abolfazl Soltani ${ }^{*}$ \\ Department of Physics, University of Birjand, Birjand, Iran \\ Email: soltani.a.physics@gmail.com
}

\begin{abstract}
As you know we do not have any exact equation for calculation Prolate Spheroid surface area. Here we will obtain this equation by analytical method. Moreover, here we will obtain the prolate spheroid equation, when the center is a focal point of prolate spheroid.

Keywords: Prolate spheroid, Ellipsoid, Oblate spheroid, Surface area

\section{Prolate Spheroid Equation}

As you know, a prolate spheroid with scales $a=a_{0}>\left(c_{0}=b_{0}\right)$ is obtained from the whole rotation of an ellipse with semi-major axis $a_{0}$ and semi minor axis $b_{0}$ around the $a_{0}$ axis. Now we want to obtain the equation of this prolate spheroid in spherical coordinates. Consider Figure 1. In this figure, a prolate spheroid will draw from the complete rotation of the blue ellipse with scales $a_{0}$ and $b_{0}$ around the $x$-axis.
\end{abstract}

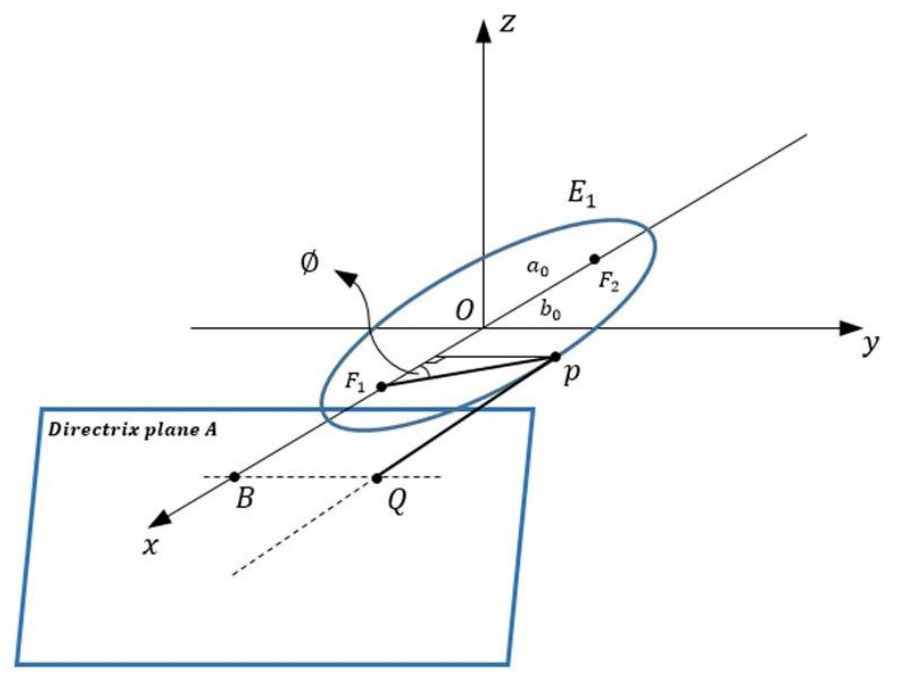

Fig. 1. As shown in the figure, the blue ellipse is on the horizontal plane $x y$. From the rotation of the blue ellipse around the $x$-axis, a prolate spheroid will build. The plane $A$ is located at a vertical distance $\frac{a}{\epsilon}$ from the origin $O$ and is perpendicular to the $x$ axis and we call it the spheroid Directrix plane.

In the ellipse in figure 1 we have [1][2]:

$$
\frac{F_{1} P}{Q P}=\frac{r}{d+r \operatorname{Sin} \theta \operatorname{Cos} \emptyset}=\epsilon_{0}
$$

Where $\epsilon_{0}$ is eccentricity of ellipse. In this equation, $Q P$ is the vertical distance from point $P$ to the spheroid directrix plane, i.e. plane $A$. This plane is perpendicular to the $x$-axis and its vertical distance to point $O$ is equal to $a_{0} / \epsilon_{0} . d$ is the distance between $F_{1}$ and $B$ (point $B$ is 
on the plane $A$ ) and we have: $d=\frac{a_{0}}{\epsilon_{0}}-\epsilon_{0} a_{0}$. Equation 1 stands for all the points $P$ on the surface of the prolate spheroid, due to we can build a prolate spheroid by rotation of the ellipse in Fig. 1 around $x$-axis. In equation $1, r \sin \theta \cos \emptyset$ is the shadow of $F_{1} P$ line on the $x$ axis and is written in spherical polar coordinates $(r . \theta . \varnothing)$ based on figure 2 , because the spheroid is a three-dimensional object. $d=\frac{a_{0}}{\epsilon_{0}}-\epsilon_{0} a_{0}$ and therefore, from 1 we have:

$$
r-r \epsilon_{0} \operatorname{Sin} \theta \operatorname{Cos} \emptyset=a_{0}\left(1-\epsilon_{0}^{2}\right)
$$

This is equation of prolate spheroid in spherical polar coordinates with center of $F_{1}$.

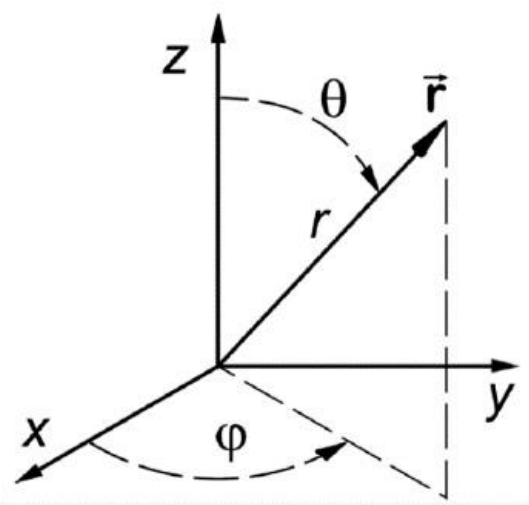

Fig. 2. Spherical polar coordinates

\section{Prolate Spheroid Surface Area}

Consider a prolate spheroid with scales $a=a_{0}>\left(c_{0}=b_{0}\right)$. In the same way as the recent preprint [3] can be shown that:

$$
\begin{gathered}
b_{0} \rightarrow 0 \text { and } a_{0}>c_{0} \gg b_{0} \Rightarrow S=4 \pi\left(\frac{a_{0}}{\sqrt{2}}\right)^{2} \\
a_{0}=b_{0}=c_{0} \Rightarrow S=4 \pi a_{0}^{2} \\
a_{0}>\left(c_{0}=b_{0}\right) \Rightarrow S=4 \pi\left(\frac{a_{0}}{\sqrt{2}^{\epsilon_{0}^{2}}}\right)^{2}
\end{gathered}
$$

In situation $b \rightarrow 0$ and $a_{0}>c_{0} \gg b_{0}$ our prolate spheroid is a flattened spheroid. In figure 3 we show a prolate spheroid with $a_{0}=5 \mathrm{~cm}, b_{0}=c_{0}=3 \mathrm{~cm}$ and its associated sphere. As you can observe its clear the surface area of our prolate spheroid is equal to its associated sphere.

$$
\epsilon_{0}=\frac{\sqrt{a_{0}^{2}-b_{0}^{2}}}{a_{0}}=\frac{\sqrt{25-9}}{5}=0.8 \Rightarrow S_{\text {Prolate spheroid }}=S_{\text {Associated sphere }}=4 \pi\left(\frac{a_{0}}{\sqrt{2}^{\epsilon_{0}^{2}}}\right)^{2}=204 \mathrm{~cm}^{2}
$$

The radius of associated sphere is $\frac{a_{0}}{\sqrt{2} \epsilon_{0}^{2}}=0.8 a_{0}=04.03 \mathrm{~cm}$ 


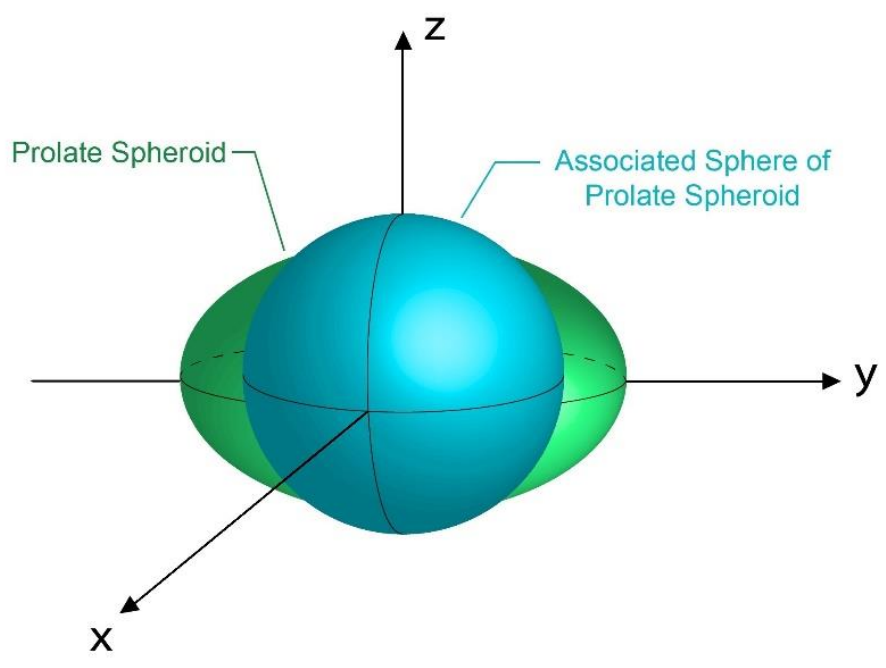

Fig. 3. A prolate spheroid and its associated sphere. Center of spheroid and sphere are on the center of cartesian coordinates system.

Therefore, the final form of prolate spheroid surface area is:

$$
S=4 \pi\left({\frac{a_{0}}{\sqrt{2}^{\epsilon_{0}^{2}}}}^{2}\right.
$$

\section{Conclusion}

In this article we used from the concept of Directrix Plane of a prolate spheroid to obtain the equation of surface area of prolate spheroid. It's clear this concept is useable for ellipsoid and subsequently oblate spheroid. Directrix Plane seems to be entering Geometry and Mathematics for the first time.

\section{References:}

[1]. Silverman, R. Calculus with Analytic Geometry (Prentice-Hall, Inc. New Jersey. 1985), pp. 628-641

[2]. Johnson, R. Calculus with Analytic Geometry (Allyn and Bacon. Inc. Boston, ed. 4), pp. 536-539

[3]. Soltani, A. Exact Equation of Ellipse Perimeter by Analytical Method, Cambridge Open Engage, DOI: https://doi.org/10.33774/coe-2022-rskdp-v3 